\title{
Promising Strategies to Support COVID-19 Vaccination of Healthcare Personnel: Qualitative Insights from the VHA National Implementation
}

\author{
Karleen F. Giannitrapani, $P h D, M P H, M A^{1,2}$, Cati Brown-Johnson, $P h D^{1,2}$ (D), \\ Natalie B. Connell, BS ${ }^{3}$, Elizabeth M. Yano, PhD, MSPH ${ }^{4,5,6}$, Sara J. Singer, PhD, MBA ${ }^{1,2}$, \\ Susan N. Giannitrapani, MSN, APRN, CCRN ${ }^{7}$, Wendy Thanassi, MD, MA ${ }^{8,2}$, and \\ Karl A. Lorenz, MD, MSHS ${ }^{1,2}$
}

\begin{abstract}
${ }^{1}$ Center for Innovation to Implementation, VA Palo Alto Health Care System, Menlo Park, CA, USA; '2Division of Primary Care and Population Health, Stanford University School of Medicine, Stanford, CA, USA; ${ }^{3}$ Emory University School of Medicine, Atlanta, GA, USA; ${ }^{4}$ Center for the Study of Healthcare Innovation, Implementation and Policy, VA Greater Los Angeles Healthcare System, Los Angeles, CA, USA; ${ }^{5}$ Department of Health Policy and Management, Fielding School of Public Health, University of California Los Angeles, Los Angeles, CA, USA; 'Department of Medicine, Geffen School of Medicine, UCLA, Los Angeles, CA, USA; ${ }^{7}$ Employee Occupational Health, Wilmington VA Medical Center, Wilmington, DE, USA; ${ }^{8}$ Occupational Health Service, VA Palo Alto Health Care System, Palo Alto, CA, USA.
\end{abstract}

BACKGROUND: In August 2021, up to 30\% of Americans were uncertain about taking the COVID-19 vaccine, including some healthcare personnel (HCP).

OBJECTIVE: Our objective was to identify barriers and facilitators of the Veterans Health Administration (VHA) HCP vaccination program.

DESIGN: We conducted key informant interviews with employee occupational health $(\mathrm{EOH})$ providers, using snowball recruitment.

PARTICIPANTS: Participants included 43 VHA EOH providers representing 29 of VHA's regionally diverse healthcare systems.

APPROACH: Thematic analysis elucidated 5 key themes and specific strategies recommended by $\mathrm{EOH}$.

KEY RESULTS: Implementation themes reflected logistics of distribution (supply), addressing any vaccine concerns or hesitancy (demand), and learning health system strategies/approaches for shared learnings. Specifically, themes included the following: (1) use interdisciplinary task forces to leverage diverse skillsets for vaccine implementation; (2) invest in processes and align resources with priorities, including creating detailed processes, addressing time trade-offs for personnel involved in vaccine clinics by suspending everything non-essential, designating process/authority to shift personnel where needed, and proactively involving leaders to support resource allocation/alignment; (3) expect and accommodate vaccine buy-in occurring over time: prepare for some HCP's slow buy-in, align buy-in facilitation with identities and motivation, and encourage word-of-mouth and hyper-local testimonials; (4) overcome misinformation with trustworthy communication: tailor communication to individuals and address COVID vaccines "in every encounter," leverage proactive institutional messaging to reinforce information, and invite bi-directional conversations about any vaccine concerns. A final overarching theme focused on learning

Received September 16, 2021

Accepted January 26, 2022

Published online March 8, 2022 health system needs and structures: (5) use existing and newly developed communication channels to foster shared learning across teams and sites.

CONCLUSIONS: Expecting deliberation allows systems to prepare for complex distribution logistics (supply) and make room for conversations that are trustworthy, bidirectional, and identity aligned (demand). Ideally, organizations provide time for conversations that address individual concerns, foster bi-directional shared decisionmaking, respect HCP beliefs and identities, and emphasize shared identities as healthcare providers.

KEY WORDS: COVID-19; vaccination; healthcare personnel; employee occupational health; qualitative research.

J Gen Intern Med 37(7):1737-47

DOI: $10.1007 / \mathrm{s} 11606-022-07439-y$

(C) This is a U.S. government work and not under copyright protection in the U.S.; foreign copyright protection may apply 2022

\section{BACKGROUND}

In August 2021, up to $30 \%$ of Americans were uncertain about taking the COVID-19 vaccine, or opposed to it. ${ }^{1-3}$ Individuals delaying vaccination reported concern related to COVID-19 vaccines rooted in rapid vaccine development, novelty of the technology, inconsistent messaging, and social media misinformation. ${ }^{4,5}$ Interestingly, despite high exposure risks, health literacy, and obligations to protect patients, some healthcare personnel (HCP) also had planned to delay or decline initial COVID-19 vaccination, citing primary concerns associated with how quickly COVID-19 vaccines were developed and approved, and personal safety (e.g., side effects) and efficacy of vaccines. ${ }^{6,7}$ Studies of HCP's delay of COVID-19 vaccines also showed that trust was a major issue, driven by distrust in government, regulatory processes, and public health experts. $^{7,8}$ Some large healthcare institutions such as the US Veterans Health Administration (VHA) were early adopters 
for both flu and COVID-19 vaccine mandates, with a HCP mandate for COVID-19 vaccines issued on July 28, 2021, and extension to volunteers and contractors on August 13, 2021, impacting $360,000 \mathrm{HCP} .{ }^{9}$ Considering this group of personnel impacted by VHA mandates, we define HCP in alignment with the Centers for Disease Control (CDC) as all onsite providers, staff, trainees, contractors, and volunteers. ${ }^{10}$

Vaccine mandates come in many shapes and sizes, informed by politico-cultural context and policy history. ${ }^{11}$ Previous studies suggest that mandate efficacy in successful $\mathrm{HCP}$ vaccination is achievable with the right resources and approaches; an international systematic review of HCP flu vaccination programs identified vaccine uptake facilitators to include mandates or active decline processes, free and accessible vaccines, education and incentive activities, organizational support over time, and dedicated personnel. ${ }^{12}$ At a positive extreme, a 10-year US medical center flu vaccine mandate that included employment termination as a consequence of noncompliance has been highly successful, with 99\% HCP immunization and positive impacts on patient safety. ${ }^{13}$ Another study comparing six national or statewide mandates recommended strategies to reduce the risk of mandate repeal: pairing mandates with public trust campaigns and exerting minimal coercion. ${ }^{11}$

Indeed, vaccine adoption strategies will continue to be needed, since mandate repeals may be an ongoing risk. ${ }^{14}$ Even in a medical context, the first year of vaccine mandating can be challenging, with potentially acute impacts on healthcare delivery and staffing. One study of a quaternary pediatric hospital found that $1.6 \%$ of HCP were placed on administrative leave in year 1 of a flu vaccine mandate program ${ }^{13}$; to give a sense of scale, translated to the VHA, at $1.6 \%$, nearly 6000 HCP would be out on leave related to COVID-19 vaccine mandates. This kind of initial vaccine resistance has already occurred in some settings. For instance, in rural New York, impacts of HCP COVID-19 vaccine resistance forced a hospital to temporarily close maternity wards in September 2021, when too many maternity staff resigned instead of taking the COVID-19 vaccine. ${ }^{15}$ Some predict that lack of mandates and/ or enforcement could become a hospital recruiting tool; in areas that already experience HCP workforce shortages, desperate need may incentivize systems to delay or not introduce mandates in hopes of recruiting and retaining HCP who might otherwise consider leaving. ${ }^{16}$ With these kinds of healthcare delivery disruption risks, even with a mandate there is still a critical role for addressing HCP concerns in order to meet immunization goals, and smooth and streamline implementation.

As expectations and regulations evolve around mandates, communication will continue to be a vital aspect of any implementation. Considering this, we could expect the role of employee occupational health $(\mathrm{EOH})$ to increase in terms of vaccine management and delivery. These new EOH roles might encompass logistics of vaccine and testing monitoring as well as communication. ${ }^{17}$ This type of role increase for
EOH would be in line with our previous study results, which showed broad EOH general role expansion as a result of COVID-19, with resulting needs revolving around infrastructure, information sharing mechanisms, staffing, leadership, and mental health support. ${ }^{18}$

In this study, we examine barriers and facilitators to vaccinating healthcare personnel in a national integrated healthcare system, specifically, the US VHA, in the context of the 20202021 effort to administer initial COVID-19 vaccines to staff, before the VHA mandate. Since the VHA is the US's only countrywide healthcare system, with this exploration of COVID-19 vaccine delivery, we aimed to illuminate challenges that large and networked systems may face, and identify promising strategies to smooth and speed vaccine deployment. Because the stakes have been so high for HCP, hospitals, and patients, we aimed to identify challenges and strategies for COVID-19 vaccine delivery to HCP in hopes that lessons learned would inform systems, strategies, and communication approaches for ongoing mandated and nonmandated COVID-19 vaccination for HCP.

\section{METHODS}

\section{Interviews}

We interviewed employee occupational health (EOH) staff from a variety of professional training groups (e.g., $\mathrm{MD} / \mathrm{DO}$, $\mathrm{PA} / \mathrm{NP}$, and $\mathrm{RN}$ ) about roles and needs for vaccine distribution to the VHA's HCP. EOH providers have perhaps the deepest view of health-related issues for healthcare workers, including vaccinations, and thus are ideal subject matter experts for key informant interviews. Semi-structured interview topics covered EOH roles generally vis-à-vis COVID-19 (see our previous publication for non-vaccination topic guide ${ }^{18}$ ), as well as vaccination planning and delivery, including EOH's role in vaccine delivery, coordination, competing demands, barriers and facilitators, management of vaccine hesitancy, and lessons learned (see Table 1 for vaccination-specific questions).

Our interview guide leveraged both implementation science frameworks and organizational theories. Specifically, the

\section{Table 1 Vaccine-Specific Interview Questions}

\footnotetext{
Vaccine-specific interview questions

Considering the COVID vaccine, what is the role of occupational health?

Who are you coordinating with?

What are your competing demands? Trade-offs - what is harder to get done?

What is working well at your facility?

What needs do you have to deliver the COVID vaccine? What are your barriers?

What do you need leadership to know about your role vis-á-vis the vaccine?

How is your site preparing people to understand what they need to know? What communication is occurring?

How are you managing any vaccine hesitancy?

What is your ideal vaccine delivery? What do you wish you could do, and how would you do it differently? What would make it go better?
} 
interview guide was informed by the Consolidated Framework for Implementation Research (CFIR), ${ }^{19}$ where we looked for barriers and facilitators to vaccine delivery. We also reviewed role-readiness literature for probes relating to what might help the frontline $\mathrm{EOH}$ providers feel more ready or prepared to support new and dynamically changing processes. ${ }^{20}$ Looking to give feedback to leaders in the national program office, we probed into what support frontline occupational health wanted and needed from various managers and leaders. ${ }^{21-24}$ Our interview guide was developed by a team with qualitative research expertise (KG, CBJ), and we conducted a run-in pilot with subject matter experts.

This study was approved by the Stanford IRB, \#56617, and funded by VA Health Services Research and Development Service (HSR\&D) C19 20-207.

\section{Data Collection}

We used a snowball sampling approach ${ }^{25}$ to attain a purposeful sample. We started with known EOH subject matter experts, and tracked variation in region and training in the sample in order to ensure diverse perspectives. Our snowball approach was sufficient in creating a varied sample, in part because we asked for additional contacts (up to three) if offered contacts were at sites we had already sampled. Potential participants were sent an email inviting them for a research interview and contacted two more times by email ( 3 total possible email contacts). During the phone interviews conducted by $\mathrm{PhD}$-trained qualitative researcher $\mathrm{CBJ}$, we obtained verbal consent for participation and audio recording. Interview questions and procedures were reviewed by an interdisciplinary research advisory team with both organizational behavior and medical expertise (SS, KL, and EY). Research notes were captured during interviews for rapid analysis, and verbatim transcripts were created from audio recordings.

\section{Participants}

Our purposive sample ${ }^{25}$ ensured key informant participants represented a diversity of geographic regions, urbanicities, site sizes, and roles. Specifically, we optimized respondent recruitment to build a sample representing a diversity of geographic regions (e.g., Northeast, Mid-Atlantic, Midwest, South, Southwest, and West), site sizes (small, midsize, and large), and urbanicities (urban, suburban, and rural). Interviews occurred over 9 months across: (1) the emergency use authorization of the first COVID-19 vaccines in December 2020, and (2) vaccine distribution as late as end of March 2021 (see Fig. 1 for interview timing).

\section{Analysis}

We derived key findings using a rapid qualitative analysis approach $^{26}$ to identify primary themes in real time from key informant interviews. Within the VHA, rapid qualitative approaches have previously successfully been used to provide real-time insights backed by high-quality rigorous research methods. ${ }^{27}$ Specifically, we used summary templates, team debriefing, consensus analysis, constant comparison, and member checking approaches to facilitate analysis and ensure validity. We summarized interviews within hours of conducting the interview using a template. We debriefed interviews as a team in weekly meetings throughout data collection, using template summaries to inform consolidated findings through a consensus analysis process informed by a constant comparison approach. ${ }^{28}$ We identified exemplary quotes from transcripts representing the major themes. Themes were validated by two EOH stakeholders as a member check. ${ }^{29}$ Our study meets the COREQ criteria for reporting qualitative research. ${ }^{30}$

\section{RESULTS}

We interviewed 43 VHA EOH providers including 22 physicians (MD/DO), 17 nurse practitioners and physician assistants (NP/PA), and 4 registered nurses (RN), from 29 of VHA's medical centers throughout the US (see Table 2). Interviews lasted approximately 30-60 min. Respondent medical centers represented a diversity of geographic regions $(9$ Northeast, 6 Mid-Atlantic, 4 Midwest, 4 South, 2 Southwest, and 4 West), site sizes (11 small, 7 midsize, and 11 large), and urbanicities (13 urban, 3 suburban, and 13 rural). Thematic analysis elucidated 5 key themes for vaccine delivery and implementation across sites that reflected logistics of distribution (supply concerns) and addressing vaccine concerns (demand concerns) in a learning health system. Supply themes included the following: (1) build interdisciplinary task forces to leverage diverse skillsets and (2) invest in processes and resources. Demand concerns included the following: (3) expect vaccine buy-in to occur over time and (4) overcome misinformation with trustworthy communication. A final overarching theme focused on learning health system needs and structures: (5) shared learnings about logistics and vaccine conversations across teams and sites. See Table 3 for overarching themes, specific strategies, and example occupational health provider quotes.

\section{Theme 1. Build Interdisciplinary Task Forces to Leverage Diverse Skillsets and Effort in Vaccine Implementation}

EOH providers perceived COVID-19 vaccination as an unprecedented, urgent problem requiring diverse skillsets (clinical, leadership/incident command, public health, bioinformatics, administrative, etc.) to plan and execute effectively. Participants noted the specific strategy of creating interdisciplinary COVID-19 vaccination teams with clear goals and roles as a major part of vaccine delivery success. Conversely, misalignment of goals and inflexible reporting structures slowed implementation and created unnecessary conflicts across siloed disciplines. At one site, managers of 


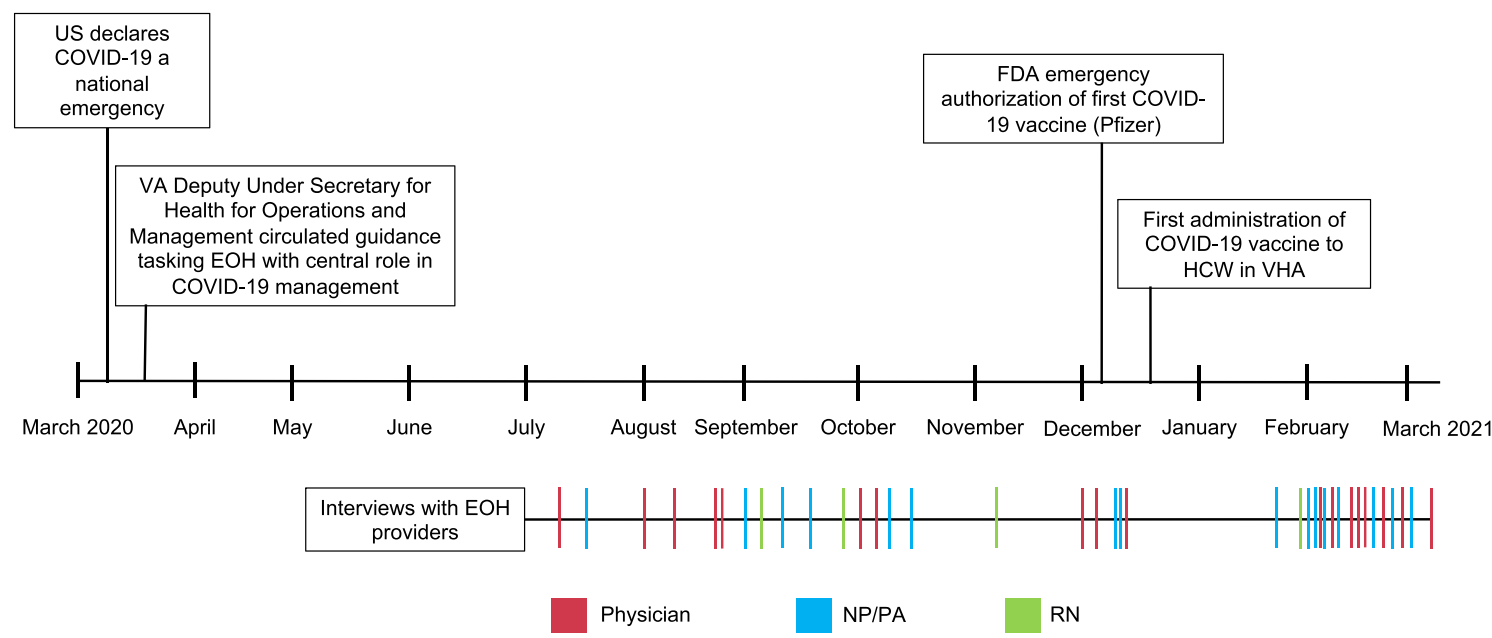

Figure 1 Relational timeline of COVID-19 in the US and distribution of EOH interviews.

four disciplines (information technology_-IT, nursing, $\mathrm{EOH}$, and management) needed to connect to reduce conflict around vaccination-related tasks. One clinician reiterated: "It's multidisciplinary... employees from social work and education have been very responsive."

VHA leveraged existing bureaucratic structures, such as the COVID-19 emergency response "incident command" site leadership teams, to rapidly deploy task forces and teams. Diverse skillsets from these COVID-19 vaccination teams helped overcome logistical barriers. Vaccine dissemination was "just not a one-person job." One physician explained, "if we could have had anything else, it would have been to have more of an employee health team when this [vaccine campaign] rolled out." Providers valued bringing together multiple disciplines, departments, and organizational levels to leverage diverse expertise, including both clinical and non-clinical parties.

Beyond interdisciplinary teamwork, integrated workflows

Table 2 Sample of Respondents and Sites

\begin{tabular}{|c|c|c|c|}
\hline \multicolumn{2}{|c|}{ Providers $(n=43)$} & \multicolumn{2}{|l|}{ Site $(n=29)$} \\
\hline \multicolumn{2}{|l|}{ Type } & \multicolumn{2}{|l|}{ Location } \\
\hline NP/PA & 17 & Northeast & 9 \\
\hline & 4 & Mid-Atlantic & 6 \\
\hline \multirow[t]{2}{*}{$\mathrm{MD} / \mathrm{DO}$} & 22 & Midwest & 4 \\
\hline & & West & 4 \\
\hline Gender & & South & 4 \\
\hline Women & 25 & Southwest & 2 \\
\hline \multirow{9}{*}{ Men } & 18 & & \\
\hline & & Size & \\
\hline & & Mid & 11 \\
\hline & & Large & 11 \\
\hline & & Small & 7 \\
\hline & & Rural/urban & \\
\hline & & Rural & 13 \\
\hline & & Urban & 13 \\
\hline & & Suburban & 3 \\
\hline
\end{tabular}

Twenty interviews occurred in July 15 to December 10, 2020. Twentythree interviews probed further into vaccine dissemination challenges and occurred between February 1 and March 10, 2021. Small sites had less than 2000 employees, midsize sites had between 2000 and 4000 employees, and large sites had over 4000 employees allowed team members to share task load and create efficiencies. An ideal process might include techs or nurses documenting vaccines, pharmacists administering vaccines, and physicians monitoring for post-vaccine adverse events. When designating stable vaccine-specific teams was not possible, providers benefitted from clearly delineated tasks that recognized interdepartmental coordination and collaboration. Through leveraging interdisciplinary expertise, vaccination teams were positioned to become "really well-oiled machine [s]," rapidly distributing vaccines to HCP.

\section{Theme 2. Invest in Processes and Align Resources with Priorities: "Focus Like a Laser"}

EOH providers reported that the COVID-19 vaccine rollout was layered on already overwhelming COVID-19 demands: "You need to be an octopus to get everything done." The need for focus was critical, particularly during early implementation. Participants noted specific supporting strategies of creating detailed processes, for instance a logistics plan to prevent wastage and allocate excess vaccine doses; addressing time trade-offs for personnel involved in vaccine clinics by suspending everything non-essential; designating process/ authority to shift personnel where needed; and proactively involving leaders to support resource allocation and alignment.

Detailed processes were critical due to vaccine complexity: "It's not like the flu shot where we can just go and grab a prefilled syringe and administer it at any point in time." One site's greatest success was what an interviewed clinician referred to as a "great process" to limit wasting precious vaccine doses: their plan involved having morning walk-in clinics to support shift-change vaccinations, and a systematic approach to sitewide communication about ad hoc opportunities to use opened but unused doses. This allowed time throughout the day to find extra vaccine recipients once a vial was open in the morning. 
Table 3 Themes and Specific Strategies to Support COVID-19 Vaccine Dissemination to Healthcare Professionals

\begin{tabular}{ll}
\hline \hline Theme & Specific strategies \\
\hline $\begin{array}{l}\text { 1. Build interdisciplinary } \\
\text { task forces to leverage }\end{array}$ & 1.1 Create interdisciplinary \\
diverse skillsets
\end{tabular}

Exemplary quotes-specific strategies

"It's not just nursing that's coordinating this vaccine administration-it's multidisciplinary. It's also education... and those employees from social work and education have been very responsive." (NP/PA)

"Everyone is working towards the same goal... amongst leadership and occupational health and infection prevention and nursing and pharmacy... Individual relationships within occupational health, individual relationships with leadership at this VA, and relationships just with the employees are the most helpful and effective things that we have going for us."

2. Invest in processes and align resources with priorities
2.1 Create detailed processes, for instance a logistics plan to prevent wastage and allocate excess vaccine doses
2.2 Address time trade-offs for personnel involved in vaccine clinics

2.3 Designate a process/authority to shift personnel where needed

2.4 Proactively involve leaders to support resource allocation and alignment

3.1 Prepare for some HCP slow buy-in

3.2 Align buy-in facilitation with identities and motivation
"We strategically opened up our [COVID-19 vaccine] walk-in hours in the morning because, when you end up with an uneven number in the morning, it's okay because you have more appointments coming in during day... We also have a communication system called Vocera ... a way to communicate the loose doses so that we can use them up as quickly as possible."

\section{"[Leadership suspended] almost} everything else. Luckily, they have suspended for a while not having to do the pre-employment physicals (competing occupational health role demands), you can do them later."

"We actually established a COVID hotline, and a lot of the providers who were no longer in clinic, a lot of them were detailed [assigned] to us, so they're now managing the COVID hotlines."

"[We needed] more staff to help, more hands to do the work. And of course, the right kind of support to get the more staff from the hospital managementyea, more leadership support...they had to approve the extra hands. That's been critical."

3. Expect and strategically prepare for vaccine buy-in occurring over time
"In terms of health literacy, what we saw as the vast majority of physicians getting vaccinated. And then, the vast majority of employees being vaccinated..."

"And I remind them [HCP] that you know what, it's not $100 \%$ proof, it's still in the test mode, but you know what, it's better than getting sick with COVID. And I said, 'you've gotta remember, we're trying to protect those vulnerable Veterans.' Same pitch I use for the flu shot. 'You know what, you and I will be fine getting through, but it's going to kill our older people, and our very
Exemplary quotes-challenges

“...it's just not a one-person job... if we could have had anything else, it would have been to have more of an employee health team when this [vaccine campaign] rolled out." "Our incident command was just like 'we need to do this', and then they were like a deer in headlights. When we went down to meet, nobody had planned anything at all...most of the incident command that was running it was non-clinical, and they were just like ducks out of water. Luckily, Primary care and Quality stepped in and fixed it, but it was a straight cluster. However, without the backing of incident command, I don't know that Quality would have gotten pulled in the pool to help give the vaccines like they did." "If you do walk-in in the afternoon and you end up with uneven numbers, then there's a higher chance that you'll end up wasting. Despite that, even though we have that set up, every afternoon, if we do have an uneven number, we do overhead messages [intercom] to the entire hospital [employees] telling them that we have extra doses, please come on down if you want a COVID vaccine"

"It has become a 24 hour per day job, where our employee health nurse practitioner now takes call, which was something we didn't previously do, and then trying to get the massive number of vaccines out as quickly as they did, was really difficult."

"I know that they may not have FTEs [full-time employees] or whatever, but we have talked so many times about if there was just one MSA-type [administrative] person who could do some scheduling and things like that, that would help incredibly."

"So as coordinator at the occupational health program, my manager was not giving me any management responsibility, that was part of the issue. I couldn't manage anyone; I couldn't really

tell anyone what to do. So that caused some problems."

"I'm just kind of old school and I never like to use new medications until they've been out for a few years...I'm never the one that jumps on. I wait."

"You also have to realize in my population, at my institution, there is vaccine fear in different ethnic groups related to cultural experience, so this was also initially present with the COVID vaccine." 
Table 3. (continued)

\begin{tabular}{|c|c|c|}
\hline Theme & Specific strategies & Exemplary quotes-specific strategies \\
\hline $\begin{array}{l}\text { 4. Overcome misinformation } \\
\text { through trustworthy } \\
\text { communication }\end{array}$ & $\begin{array}{l}\text { 4.1 Tailor communication to } \\
\text { individuals and address COVID } \\
\text { vaccines "in every encounter" }\end{array}$ & $\begin{array}{l}\text { young. So, think about that... Do you } \\
\text { want to bring this home to your kids?" } \\
\text { "The buy-in came because people [in } \\
\text { our facility] were getting it and not } \\
\text { having side effects, and then it was sort } \\
\text { of like a tsunami with everybody- } \\
\text { "Okay, well, you got yours, I'll get } \\
\text { mine'." } \\
\text { "A long trail, a slow trickle of } \\
\text { employees that's been pretty steady } \\
\text { every week since the first push. And } \\
\text { on-going with } 30,40 \text { people interested } \\
\text { getting vaccinated per week, after they } \\
\text { have witnessed the lack of side effects } \\
\text { with the vaccines." } \\
\text { "Anecdotally, there is a lot of } \\
\text { hesitancy and I think kind of taking it } \\
\text { one person at a time in our } \\
\text { interactions, and you know, if someone } \\
\text { comes in to get a COVID swab, I think } \\
\text { we should be talking about the vaccine } \\
\text { in every encounter if possible." }\end{array}$ \\
\hline
\end{tabular}

Exemplary quotes - challenges

3.3 Encourage word-of-mouth accounts and hyper-local testimonials ndividuals and address COVID through trustworthy communication
4.2 Leverage proactive institutional messaging (e.g., town halls, email campaign, educational presentations, Q\&A sessions) to reinforce information access and clear communication

4.3 Invite open bi-directional conversations about vaccines

5.1 Create infrastructure for cross-site learning and information sharing

\section{"I think it is a tremendous} responsibility to get the word out, to market, answer everyone's questions in the form of town halls, and get as many employees vaccinated for COVID as possible."
5. Foster sharing and learning across teams and sites using existing and newly developed communication channels
[Be willing to have the conversation]: "I can tell you what literature I've seen, I can tell you what I've found, I can tell you what folks have experienced. Bottom line, it is your call. The recommendation is this. I can see if I can find additional literature to help you make that decision, but it is ultimately your call."

"The one thing that has probably been really helpful with our VISN [Region] is we have a fairly strong commitment with each other to doing a monthly, or actuallyevery two weeks for a little while, telephone conference...discussing what are we facing, how is a particular area doing. Like, for us, [SiteA] was a site that received the vaccine first... So they're within my VISN and I know their occupational health person, who is on the phone call and is like, 'hey, this is what we're doing down here'."
"It takes a bit of mindset sometimes when people are kind of fixated at something, so if you start to let them have their thought processes and why they think what they think. And I'm honest with them, I'm like, 'You know what, I was one of the hesitancy ones, guys. If it was [one vaccine], I wouldn't have signed up for it. [Another vaccine] - I liked it, and this is what I liked about it. This is why I think it's a good thing'."

"We've had afew people here and there who don't want it for whatever reason.You know, some young ladies, you know, worry about their fertility, we have a couple pregnant people who don't want to get it while they're pregnant, and things like that."

"We have a very diverse employee population, and many of the minorities who suffered a lot through the COVID pandemic are adversely affected by this misinformation, maybe due to their ethnicity or culture and past experience, they're more fearful of getting vaccinated due to their past experience, so I think there's a lot to overcome there as well."

"Some of the stuff that this person was reading online, I'm just very surprised at what's out there and unfortunately it has steered some people away from it [getting vaccinated]."

"I think where we're missing is maybe a medium there where the public can access the information easily and have them understand it and not have it be overshadowed by stuff that they're reading on social media or the internet... I wish there was you know maybe more, I have access to all the journals and efficacy rates of the different vaccines."

"Someone had asked me, you know, 'are there are any microchips in the vaccine,' and at first I thought the person was totally joking but actually they were dead serious, and then, you know, I stepped back and answered her question, you know, telling her exactly what we know based on the science."

"Would be nice to have a regional or national forum for Occ healthin which people can share their ideas... you know, I didn't have anybody I could call and say, 'Hey, you know, how do you do this? How do you do that?'...I mean we had to think outside the box to come up with a solution, but it would have been so nice if people brainstormed together." 
Frontline staff expressed appreciation when leaders supported resource allocation and were available to "approve" extra hands and "suspend" other activities, proactively addressing time trade-offs. Specifically, everything "worked better" when there was designated process/authority to reassign personnel to high-need situations. Indeed, proactively involving leaders in supporting resource alignment was "critical": "the right kind of support... was more leadership support... They had to approve the extra hands." Conversely, when authority for assigning vaccine tasks was unclear, problems arose. A clinician reported, "My manager was not giving me any management responsibility...I couldn't really tell anyone what to do. So that caused some problems."

\section{Theme 3. Expect and Strategically Prepare for Vaccine Buy-In Occurring Over Time}

Even among EOH providers, a few reported "wait[ing]" on vaccination, in addition to noting some HCP reluctance. $\mathrm{EOH}$ providers perceived vaccine reluctance to be related to various factors beyond mistrust, including "health literacy" and "ideology." EOH providers noted "how politicized COVID has become," and how "it's hard to disentangle the political aspects from the public health aspects."

Specific strategies to account for some deliberation about vaccines included preparing for some HCP's slow buy-in, aligning buy-in facilitation with identities and motivation, and encouraging word-of-mouth and hyper-local testimonials. EOH providers disclosed that expecting slow buy-in among some HCP primed them to think strategically about facilitating vaccine acceptance over time. Important approaches to facilitating HCP buy-in included being alert to individual employee concerns and background, and aligning motivations with identities. For individual employee concerns, $\mathrm{EOH}$ providers reflected on belief systems and/or personal experiences that might have guided vaccination choice, allowing them to better meet HCP where they might be at in their thinking: "maybe due to their ethnicity or culture and past experience, they're more fearful of getting vaccinated." By attempting to understand HCP perspectives, EOH providers could foster vaccination buy-in through conversations that aligned HCP personal motivations with important identities, such as that of being a parent (e.g., "[Consider getting vaccinated because you don't] want to bring this home to your kids").

Hyper-local testimonial and word-of-mouth accounts from EOH providers and HCP peers were also strategies to support acceptance: "The buy-in came because people were getting it and not having side effects, and then it was sort of like a tsunami... 'Okay, well you got yours; I'll get mine'." A few $\mathrm{EOH}$ providers emphasized the importance of sharing their own personal story - reasons why they had chosen to receive the vaccine (e.g., to see elderly parents, because they had witnessed even very careful co-workers contract it at work, because someone they knew personally had died) and their experience with vaccination. EOH staff reported that with such efforts, HCP who were previously reluctant chose vaccination. One clinician described the process: "The vast majority of employees [got] vaccinated [right away], followed by a long trail, a slow trickle of employees that's been pretty steady."

\section{Theme 4. Overcome Misinformation Through Trustworthy Communication}

EOH providers noted feeling that they were "swimming upstream" against massive amounts of misinformation online, particularly on social media or targeting specific groups: "I think there's a terrible misinformation campaign on the internet...many of the minorities who suffered a lot through the COVID pandemic are adversely affected by this misinformation."

To address vaccine reluctance related to misinformation, EOH providers enumerated specific strategies that embrace trustworthy communication and promote trust-building, including tailoring communication to individuals and addressing COVID vaccines "in every encounter"; leveraging proactive institutional messaging (e.g., town halls, Q\&As) to reinforce information access and clear communication; and inviting open bi-directional conversations about vaccines. One EOH provider noted, "take it one person at a time" in tailored communication with individuals, and suggested building trust by addressing personal HCP concerns and fears "in every encounter": "if someone comes in to get a COVID swab, I think we should be talking about the vaccine." On a larger scale, EOH providers had a strategy of communicating clear institutional messages for HCP through town halls, email campaigns, educational presentations, and/or Q\&A sessions that also allowed bi-directional communication ("We had several townhalls to allow employees to ask questions."). $\mathrm{EOH}$ providers recommended expecting decisions to occur over time and through multiple bi-directional communications, including invited "shared decision-making" conversations that empowered HCP.

\section{Theme 5. Foster Sharing and Learning Across Teams and Sites}

EOH providers reported exhaustion from the constant experience of "reinventing the wheel," resulting in the desire to learn from the ongoing experiences of other staff at their site and beyond: "it would have been so nice if people brainstormed together."

EOH providers reported a primary strategy for shared learnings, which was to create or leverage infrastructure for crosssite learning and information sharing. Within their own sites, EOH providers cited email campaigns and memos from local site champions (who represented various disciplines and typically aided local vaccination campaigns) as key local sources for sharing information. The VHA also supported EOH teleconferences across multiple sites which were a well-received 
innovation to foster shared learnings about logistics as well as vaccine responses and strategies for uptake.

Among EOH providers, a VHA-wide listserv was used to raise questions and share information about relevant topics, including COVID-19 vaccine launch. As one respondent noted, "the listserv has been a big advantage...they can ask a question, and anybody can answer those questions."

\section{DISCUSSION}

This novel study leveraged employee occupational health $(\mathrm{EOH})$ provider subject matter expertise from a national health system to explore barriers and facilitators to COVID-19 vaccine delivery, including vaccine uptake and buy-in by healthcare professionals (HCP). Since the VHA is the US's only countrywide healthcare system, this exploration of COVID-19 vaccine delivery illuminates challenges that large and networked systems may face, and identifies promising strategies to smooth and speed vaccine deployment, which could be applied to settings with or without vaccine mandates. Frontline $\mathrm{EOH}$ providers identified vaccine implementation strategies and barriers that were operational/logistical as well as interpersonal. Themes around implementation and promotion of COVID-19 vaccination included leveraging interdisciplinary teams, aligning process and resources, strategically preparing for slow buy-in among some HCP, prioritizing trustworthy communication, and facilitating shared learnings across sites.

Providers in our study observed two groups of HCP, in line with diffusion of innovations ${ }^{31}$ : there were on one hand early adopters - an early "tsunami" of adoption; conversely, EOH providers reported that some HCP were hesitant. For the early "tsunami," the leading edge of a standard diffusion of innovation curve was perhaps compressed by a compelling need for rapid adoption to prevent unnecessary loss of life. The front of the curve was compressed, but without ongoing compelling needs for rapid adoption (e.g., mandates or new highly contagious variants), we could predict regression to a more standard diffusion of innovations curve ${ }^{31}$ : a long "slow trickle" of employees embracing vaccination over time. As with any innovation, particularly one that is complex and early in terms of stage of evidence, we need real implementation strategies for later adopters; having the expectation that some HCP will delay vaccination gives implementers, facility leaders, and policy makers an appropriate orientation of needing to continue to allocate resources, time, and expertise to adoption facilitation over time.

As of writing, vaccine mandates are haphazardly in place, even in medical contexts where unintentional COVID-19 exposure may harm vulnerable patients. Our results point to four aspects needed to increase buy-in for those HCP who may still be deliberating. Organizations would ideally (1) provide adequate time for HCP to have conversations with informed providers, such as those in $\mathrm{EOH}$ roles; those conversations would (2) address individual concerns and foster bi-directional shared decision-making. Further, conversations and communication with HCP who are still deliberating would (3) respect diverse beliefs and identities of HCP, and (4) be delivered with an emphasis on shared HCP identity.

1. Employee occupational health (EOH) providers need adequate time for conversations. Our study revealed themes around a suite of strategies related to vaccine promotion that rely strongly on interpersonal interactions, including word-of-mouth peer accounts of taking the vaccine without major side effects; hyper-local testimonials, tailored to specific identities; and $\mathrm{EOH}$ providers sharing their own personal stories. EOH providers reported needing additional time to support communication during interpersonal interactions, facilitate vaccine buy-in, and solidify their role as trusted provider. This trust-building deserves dedicated attention and time; a study examining concepts of trust and hesitancy in the context of flu vaccine uptake documented that the impact of trust on vaccine acceptance operates independent of hesitancy. ${ }^{32}$ Furthermore, even in situations of high vulnerability for employees, trust in $\mathrm{EOH}$ providers can increase with more contact between employees and providers. ${ }^{33}$ An American Board of Internal Medicine study of physician trust found that post-COVID, $30 \%$ of physicians reported diminished trust in healthcare institutions, but retained high levels of trust in other healthcare personnel. ${ }^{34}$ This suggests that EOH providers might emphasize personal connections with VHA employees around shared HCP identities, and de-emphasize their role as representatives of a specific health institution. Moving forward, healthcare systems need to ensure increased staffing of $\mathrm{EOH}$ or other appropriate roles so that these providers have the flexibility to engage in in-depth conversations about vaccination with $\mathrm{HCP}$ who may be uncertain about COVID-19 vaccines.

2. Conversations with HCP need to address individual concerns and foster bi-directional shared decisionmaking. Surveys have emphasized varying approaches to facilitating vaccine acceptance through the mechanisms of shared decision-making - essentially meeting people where they are. ${ }^{3,35}$ Indeed, vaccine delay can reflect dissent, ${ }^{36}$ deliberation, ${ }^{37}$ distrust, ${ }^{38}$ and/or indifference, ${ }^{39}$ each of which would require its own facilitation approach. ${ }^{40}$ For instance, a March 2021 survey revealed that among those who wanted to wait and see before receiving the COVID-19 vaccine, education about efficacy in preventing hospitalization and death promoted vaccine acceptance. ${ }^{3}$

3. Conversations with HCP would benefit from considering and respecting their beliefs and identities. Our findings highlight the impact of the diverse identities of HCP which span professional and personal identities - on 
COVID-19 vaccine decision-making. HCP are situated in communities with distinctive belief systems and communal identities, both inside and outside their workplace. Social psychology literature emphasizes links between context and cognition in the concept of "situated cognition" ${ }^{41}$; relevant to vaccine buy-in, individuals' decisions are informed by context. ${ }^{41}$ Further, HCP may embody multiple identities simultaneously, and even in competition. ${ }^{42}$ Planning for some slow buy-in, systems and implementers need to be truthful and persuasive. Key elements include identity-aligned communication coupled with identity-based motivation (i.e., you are HCP and HCP get vaccinated to protect vulnerable patients), ${ }^{43}$ and community- and systemlevel communication approaches to combat systemic misinformation. ${ }^{40}$

4. Communication with HCP may benefit from EOH providers emphasizing their shared identity as health care personnel. This aligns with results from other surveys which have suggested that vaccine education may be even more persuasive if delivered by providers with concordant identities, e.g., same race/ethnicity, same community. ${ }^{44}$ Our study demonstrated that the HCP identity may be a powerful shared identity that EOH providers can emphasize in discussions with other employees. EOH providers in our study also spoke to the value of overcoming misinformation and building trust through educational campaigns, which could be delivered by multiple members of a multidisciplinary vaccine implementation team to increase the likelihood that HCP would perceive the messages as coming from someone who is identity-concordant. From public health campaigns, we could predict that education would be more effective in promoting vaccine acceptance among HCP if backed by anecdotes from providers with concordant identities, such as those rooted in race/ethnicity, background, or even training (nurses addressing nurses, etc.). As spread of transmissible variants threatens regions or populations where vaccination is not pervasive,${ }^{45}$ fostering vaccine acceptance while being conscious of aligning not only the message content but also the message delivery attuned to HCP identities is of critical importance.

Strengths and Limitations. Our study has the following limitations. First, we focused on $\mathrm{EOH}$ providers and thus do not have direct perspectives from HCP who delayed or declined vaccination. However, EOH providers were in the position to comment about their experiences working with many and diverse $\mathrm{HCP}$ as well as to represent a wide range of settings. Secondly, although we have strong recommendations to make time for shared decision-making conversations, we did not directly observe or record conversations to know how these conversations might actually influence HCP perspectives. Other studies should deeply examine the perspective of HCP who are hesitant about or even resistant to vaccines. One such study examining motivations for HCP COVID-19 vaccination delay complements our perspective with nuance around the reasons why HCP may be hesitant, including changeable factors such as the desire for longer-term safety and efficacy data, as well as more static beliefs, such as the belief in the right to make individual choices. ${ }^{46}$ Observation of successful conversations pertaining to the COVID-19 vaccine would be ideal and could be a future step in this work. The major strength in this work is the breadth of national perspective and experience captured herein. Again, as the only national health system in the US, the perspective of individuals across the VHA represents an important, immediately generalizable sample of national representation with themes that held salience across multiple settings. We also hope that a strength of this work resides in the practical recommendations delivered that can be immediately used to promote up-to-date vaccination among HCP.

\section{CONCLUSIONS}

Like any innovation, COVID-19 vaccine uptake may follow an adoption curve, including individuals who delay vaccination and deliberate over time. Strategies to facilitate vaccine buy-in that address individual and communal identities may be supportive of adoption; hyper-local testimonials were helpful in aligning the HCP community in one facility, spurring vaccine acceptance. Even when COVID-19 vaccination is mandatory, encouraging healthcare personnel to choose vaccination is critical; expecting some gradual acceptance will allow providers and systems to focus efforts, prepare logistically, and have trustworthy conversations that are bidirectional. To support HCP choice of vaccination as well as future large-scale changes/innovations in healthcare, ideally organizations would (1) provide time for conversations (about vaccines), and those conversations would (2) address individual concerns and foster bi-directional shared decision-making, (3) respect diverse beliefs and identities of $\mathrm{HCP}$, and (4) emphasize identity concordance through shared identities as healthcare providers.

Acknowledgements: We would like to acknowledge the participation of other project team members, Cheyenne DeShields BS MSSA and Matthew McCaa MSOT, as well as leaders from VHA occupational health programs. We would like to thank Lisa Rubenstein MD and Steven Asch MD for conceptual comments and feedback. We want to especially thank all the VHA occupational health providers who took the time to participate in interviews and give feedback on earlier versions of the work.

Corresponding Author: Karleen F. Giannitrapani, $\mathrm{PhD}, \mathrm{MPH}, \mathrm{MA}$; Center for Innovation to Implementation, VA Palo Alto Health Care System, Menlo Park, CA, USA (e-mail: Karleen@stanford.edu). 
Funding Veterans Affairs Health Services Research and Development Rapid Response COVID-19 Pilot: A National Needs Assessment for the role of Employee Occupational Health during COVID-19 (C19 20207: PI Giannitrapani); Dr. Yano's time was supported by a Research Career Scientist award (RCS 05-195); Dr. Giannitrapani's time was supported by HSR\&D Career Development Award (CDA-2 19-075).

\section{Declarations:}

Ethical Considerations and Disclosures: Our work was approved by the VA Palo Alto/Stanford IRB (\#56617).

Conflict of Interest: The authors declare that they do not have any conflicts of interest.

\section{REFERENCES}

1. Hamel L, Krizinger A, Lopes L, Kearney A, Sparks G, Brodie M. KFF COVID-19 Vaccine Monitor: January 2021. KFF COVID-19 Vaccine Monitor. Published January 22, 2021. Accessed August 28, 2021. https://www.kff.org/coronavirus-covid-19/report/kff-covid-19-vaccinemonitor-january-2021/

2. Dubé E, Laberge C, Guay M, Bramadat P, Roy R, Bettinger J. Vaccine hesitancy: an overview. Hum Vaccin Immunother. 2013;9(8): 1763-1773. doi:https://doi.org/10.4161/hv.24657

3. Huetteman E. Covid Vaccine Hesitancy Drops Among All Americans, New Survey Shows. Kaiser Health News. Published March 30, 2021. Accessed August 23, 2021. https://khn.org/news/article/covid-vaccinehesitancy-drops-among-americans-new-kff-survey-shows/

4. Loomba S, de Figueiredo A, Piatek SJ, de Graaf K, Larson HJ. Measuring the impact of COVID-19 vaccine misinformation on vaccination intent in the UK and USA. Nat Hum Behav. 2021;5(3):337-348. doi:https://doi.org/10.1038/s41562-021-01056-1

5. Wouters OJ, Shadlen KC, Salcher-Konrad M, et al. Challenges in ensuring global access to COVID-19 vaccines: production, affordability, allocation, and deployment. Lancet. 2021;397(10278):1023-1034. doi:https://doi.org/10.1016/S0140-6736(21)00306-8

6. Shekhar R, Sheikh AB, Upadhyay S, et al. COVID-19 Vaccine Acceptance among Health Care Workers in the United States. Vaccines. 2021;9(2):119. doi:https://doi.org/10.3390/vaccines9020119

7. Lucia VC, Kelekar A, Afonso NM. COVID-19 vaccine hesitancy among medical students. $J$ Public Health (Oxf). Published online December 26 , 2020:fdaa230. https://doi.org/10.1093/pubmed/fdaa230

8. Li M, Luo Y, Watson R, et al. Healthcare workers' (HCWs) attitudes and related factors towards COVID-19 vaccination: a rapid systematic review. Postgraduate Medical Journal. Published online June 30, 2021. https:// doi.org/10.1136/postgradmedj-2021-140195

9. Shivaram D. Nearly 300,000 More Federal Health Workers Are Ordered To Be Vaccinated. NPR. https://www.npr.org/sections/coronavirus-liveupdates /202 1/08/12/1027075086/covid-vaccine-hhs-va-federalhealth-workers-vaccination-mandate. Published August 12, 2021. Accessed August 28, 2021.

10. Burrer SL, de Perio MA, Hughes MM, et al. Characteristics of Health Care Personnel with COVID-19 - United States, February 12-April 9, 2020. MMWR Morb Mortal Wkly Rep. 2020;69(15):477-481. https://doi. org/10.15585/mmwr.mm6915e6

11. Attwell K, Navin MC, Lopalco PL, Jestin C, Reiter S, Omer SB. Recent vaccine mandates in the United States, Europe and Australia: A comparative study. Vaccine. 2018;36(48):7377-7384. doi:https://doi. org/10.1016/j.vaccine.2018.10.019

12. Paterson P, Meurice F, Stanberry LR, Glismann S, Rosenthal SL, Larson HJ. Vaccine hesitancy and healthcare providers. Vaccine. 2016;34(52):6700-6706. doi:https://doi.org/10.1016/j.vaccine.2016.10. 042

13. Kitt E, Burt S, Price SM, et al. Implementation of a Mandatory Influenza Vaccine Policy: A 10-Year Experience. Clinical Infectious Diseases. 2021;73(2):e290-e296. doi:https://doi.org/10.1093/cid/ciaa782

14. Clark D, Tsirkin J. Senate passes resolution to repeal Biden vaccine mandate, with help of two Democrats. NBC News. https://www. nbcnews.com/politics/congress/senate-passes-resolution-defund-repeal-biden-vaccine-mandate-n1285636. Published December 8, 2021. Accessed January 14, 2022

15. Vendel C. Hospital to stop delivering babies after staff resigns instead of getting vaccinated. pennlive. Published September 10, 2021. Accessed
September 14, 2021. https://www.pennlive.com/news/2021/09/hospital-to-stop-delivering-babies-after-staff-resigns-instead-of-getting-vaccinated.html

16. Weber L. Lack of a Vaccine Mandate Becomes Competitive Advantage in Hospital Staffing Wars. Kaiser Health News. Published online August 31, 2021. Accessed September 14, 2021. https://khn.org/news/article/ covid-hospital-staff-shortage-lack-of-mandate-competitive-advantageworker-recruitment-retention/

17. Wang TL, Jing L, Bocchini JA. Mandatory influenza vaccination for all healthcare personnel: a review on justification, implementation and effectiveness. Current Opinion in Pediatrics. 2017;29(5):606-615. doi:https://doi.org/10.1097/MOP.0000000000000527

18. Brown-Johnson C, McCaa MD, Giannitrapani S, et al. Protecting the healthcare workforce during COVID-19: a qualitative needs assessment of employee occupational health in the US national Veterans Health Administration. BMJ Open. Published online 2021:e049134-e049134.

19. Damschroder LJ, Aron DC, Keith RE, Kirsh SR, Alexander JA, Lowery JC. Fostering implementation of health services research findings into practice: a consolidated framework for advancing implementation science. Implementation Science. 2009;4(1):50. doi:https://doi.org/10. 1186/1748-5908-4-50

20. Holt DT, Helfrich CD, Hall CG, Weiner BJ. Are You Ready? How Health Professionals Can Comprehensively Conceptualize Readiness for Change. J Gen Intern Med. 2010;25(Suppl 1):50-55. doi:https://doi.org/10.1007/ s11606-009-1112-8

21. Giannitrapani KF, Rodriguez $\mathbf{H}$, Huynh $\mathbf{A K}$, et al. How middle managers facilitate interdisciplinary primary care team functioning. Healthc (Amst). 2019;7(2):10-15. doi:https://doi.org/10.1016/j.hjdsi. 2018.11.003

22. Giannitrapani KF, Glassman PA, Vang D, et al. Expanding the role of clinical pharmacists on interdisciplinary primary care teams for chronic pain and opioid management. BMC Family Practice. 2018;19(1):107. doi:https://doi.org/10.1186/s12875-018-0783-9

23. Giannitrapani KF, Soban L, Hamilton AB, et al. Role expansion on interprofessional primary care teams: Barriers of role self-efficacy among clinical associates. Healthc (Amst). 2016;4(4):321-326. https://doi.org/ 10.1016/j.hjdsi.2016.03.004

24. W B, Joanna J. Health Care Management During Covid-19: Insights from Complexity Science. NEJM Catalyst Innovations in Care Delivery. Published online October 9, 2020. Accessed August 23, 2021. https:// catalyst.nejm.org/doi/full/10.1056/CAT.20.0541

25. Bernard HR. Research Methods in Anthropology: Qualitative and Buantitative Approaches. 4th ed. AltaMira Press; 2006.

26. Brown-Johnson C, Safaeinili N, Zionts D, et al. The Stanford Lightning Report Method: A comparison of rapid qualitative synthesis results across four implementation evaluations. Learn Health Syst. 2019;4(2). https:// doi.org/10.1002/lrh2.10210

27. Hamilton AB, Brunner $\mathbf{J}$, Cain $\mathbf{C}$, et al. Engaging multilevel stakeholders in an implementation trial of evidence-based quality improvement in VA women's health primary care. Behav Med Pract Policy Res. 2017;7(3):478-485. doi:https://doi.org/10.1007/s13142-017-0501-5

28. Corbin J, Strauss A. Basics of Qualitative Research: Techniques and Procedures for Developing Grounded Theory. 3rd edition. SAGE Publications, Inc; 2007.

29. Birt L, Scott S, Cavers D, Campbell C, Walter F. Member Checking: A Tool to Enhance Trustworthiness or Merely a Nod to Validation? Qualitative Health Research. Published online June 22, 2016. doi:https://doi.org/10.1177/1049732316654870

30. Tong A, Sainsbury P, Craig J. Consolidated criteria for reporting qualitative research (COREQ): a 32-item checklist for interviews and focus groups. International Journal for Quality in Health Care. 2007;19(6):349-357. doi:https://doi.org/10.1093/intqhe/mzm042

31. Rogers EM. Diffusion of Innovations, 4th Edition. Simon and Schuster; 2010.

32. Guinn SC, Jamison AM, An J, Hancock GR, Freimuth VS. Measuring vaccine hesitancy, confidence, trust and flu vaccine uptake: Results of a national survey of White and African American adults. Vaccine. 2019;37(9):1168-1173. doi:https://doi.org/10.1016/j.vaccine.2019.01. 033

33. Plomp HN, Ballast N. Trust and vulnerability in doctor-patient relations in occupational health. Occupational Medicine. 2010;60(4):261-269. doi:https://doi.org/10.1093/occmed/kqq067

34. Public \& Physician Trust in the U.S. Health Care System. Building Trust. Accessed January 6, 2022. https://buildingtrust.org/public-physiciantrust-in-the-u-s-health-care-system/ 
35. Thompson D. The Surprising Key to Combatting Vaccine Refusal. The Atlantic. Published February 28, 2021. Accessed August 23, 2021. https://www.theatlantic.com/ideas/archive/2021/02/vaccine-hesitancy-isnt-just-one-thing/618164/

36. Koltai K. Vaccine information seeking and sharing: How private Facebook groups contributed to the anti-vaccine movement online. AoIR Selected Papers of Internet Research. Published online October 5, 2020. doi:https://doi.org/10.5210/spir.v2020i0.11252

37. Grumbach K, Judson T, Desai M, et al. Association of Race/Ethnicity With Likeliness of COVID-19 Vaccine Uptake Among Health Workers and the General Population in the San Francisco Bay Area. JAMA Intern Med 2021;181(7):1008-1011. doi:https://doi.org/10.1001/jamainternmed. 2021.1445

38. Corbie-Smith G. Vaccine Hesitancy Is a Scapegoat for Structural Racism JAMA Health Forum. 2021;2(3):e210434. https://doi.org/10.1001/ jamahealthforum.2021.0434

39. Mann DL. Now That We Have an Effective Vaccine for COVID-19, Will It Also Inoculate Us Against the Virus of Indifference? JACC Basic Transl Sci. 2021;6(1):86-87. doi:https://doi.org/10.1016/j.jacbts.2020.12.001

40. Burgess RA, Osborne RH, Yongabi KA, et al. The COVID-19 vaccines rush: participatory community engagement matters more than ever Lancet. 2021;397(10268):8-10. doi:https://doi.org/10.1016/S01406736(20)32642-8

41. Brown JS, Collins A, Duguid P. Situated Cognition and the Culture of Learning. Educational Researcher. 1989;18(1):32-42. doi:https://doi.org/ 10.3102/0013189X018001032
42. Gaither SE. The multiplicity of belonging: Pushing identity research beyond binary thinking. Self and Identity. 2018;17(4):443-454. doi:https://doi.org/10.1080/15298868.2017.1412343

43. Oyserman D, Destin M. Identity-based motivation: Implications for intervention. Couns Psychol. 2010;38(7):1001-1043. doi:https://doi. org/10.1177/0011000010374775

44. Olanipekun T, Abe T, Effoe V, et al. Attitudes and Perceptions Towards Coronavirus Disease 2019 (COVID-19) Vaccine Acceptance Among Recovered African American Patients. J Gen Intern Med. 2021;36(7):2186-2188. doi:https://doi.org/10.1007/s11606-02106787-5

45. Mahase E. Delta variant: What is happening with transmission, hospital admissions, and restrictions? BMJ. 2021;373:n1513. doi:https://doi. org/10.1136/bmj.n1513

46. Mahoney M, Winget M, Brown-Johnson C, et al. Gearing up for a COVID-19 Vaccine Requirement: A Mixed Methods Study of COVID-19 Vaccine Confidence among Health Care Workers at a large Academic Medical Center Megan Mahoney MD1-2, Marcy Winget PhD1, Cati Brown-Johnson PhD1, Lindsay de Borba3, Darlene Veruttipong1, Jacklyn Luu4, David Jones2, Bryan Bohman1-2, Stacie Vilendrer MD1. Journal of Healthcare Management. in press.

Publisher's Note: Springer Nature remains neutral with regard to jurisdictional claims in published maps and institutional affiliations. 